\title{
IR Training in the United States
}

\author{
John A. Kaufman, MD, MS ${ }^{1}$ \\ ${ }^{1}$ Dotter Interventional Institute, Oregon Health and Science \\ University Hospital, Portland, Oregon, United States \\ J Clin Interv Radiol ISVIR 2017;1:68.
}

\begin{abstract}
Address for correspondence John A. Kaufman, MD, MS, Dotter Interventional Institute, Frederick S. Keller Professor of Interventional Radiology, Oregon Health and Science University Hospital, Mail Code L-605, 3181 SW Sam Jackson Pk Rd, Portland, OR 97239 (e-mail: kaufmajo@ohsu.edu).
\end{abstract}

In 2012 the member boards of the American Board of Medical Specialties (ABMS) approved the transition of Interventional Radiology (IR) from a subspecialty of Diagnostic Radiology (DR) to a primary specialty of the American Board of Radiology (ABR) equivalent to DR, General Surgery, Internal Medicine, and the other specialties. As part of this change in status, the ABMS approved a new training pathway that would lead to a certificate including two competencies: Interventional Radiology and Diagnostic Radiology (the IR/DR certificate). Subsequently, in 2015, the Accreditation Council of Graduate Medical Education (ACGME) approved the first training programs that would lead to an IR/DR certificate. Unlike fellowships, which required completion of a DR residency prior to training, one form of these new training programs allows medical students to enter a pathway leading to an IR/DR certificate immediately after graduation. Termed "Integrated Residencies", the first were rolled out in 2016. As of March of 2017, nearly 120 medical students were matched directly into these training programs; remarkably, more than 450 medical students applied. As a frame of reference, IR fellowships in the United States can only accommodate roughly 220 trainees per year.

The IR/DR certificate and the training programs leading to this are only the latest steps in over 20 years of evolution of training in IR. The need to provide training in nonprocedural patient care has driven these changes and ultimately led to the recognition of IR as a true specialty by the ABMS. However, the process was difficult, slow, and fraught with setbacks. The initial changes occurred in 2000, when the Society of Interventional Radiology proposed a Clinical Pathway in IR training that included 2 years of IR rotations. This had very limited uptake by DR residencies and was followed in 2005 by a special ABR program termed the "DIRECT pathway" (Diagnostic and Interventional Radiology Enhanced Clinical Training and Certification). This program was intended as an alternate pathway into IR for trainees from other specialties after second postgraduate year 2, and shortened the DR portion of training by 1 year. This also had limited uptake by DR residencies.

Within a year of the DIRECT pathway, work began on what would ultimately become the IR/DR certificate and the IR residency. Over period of almost 7 years, multiple proposals were developed, vetted, modified, and discarded. Ultimately a widely supported proposal was approved by the ABMS in 2012. They process only became successful once there was input from and demonstration of wide support within the DR community. In many ways the IR/DR certificate was the result of a classic change management process, which required delineation of a clear and urgent need for change; identification and accommodation of key stakeholders; securing the initial support of the most influential stakeholders; and careful delivery of a consistent and convincing message.

IR training in the United States is now in the transition phase, with IR fellowships overlapping with residencies available to medical students. By 2020 the fellowships will be phased out, and training will be either through integrated residencies (enter from medical school: internship, 3 years of DR, 2 years of IR) or independent residencies (enter from DR residency much like current fellowships: 2 additional years of IR unless the resident has completed an early specialization in IR (ESIR) curriculum during DR residency that permits "advanced placement" into the last year of the independent IR residency). This inherent flexibility accommodates the needs of DR residents who decide late to enter IR training and DR residencies that do not have an associated pathway leading to IR/DR certification. This flexibility is further enhanced by the co-location of the large majority of the DR and IR training programs under one roof - the DR department. Regardless of the pathway, all IR training now leads to the IR/DR specialty certificate in the United States.
DOI https://doi.org/ $10.1055 / \mathrm{s}-0037-1603449$. ISSN $0000-0000$
Copyright ( 2017 by Indian Society of Vascular and Interventional Radiology
License terms

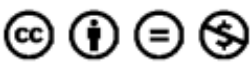

